\title{
NEES Multidirectional Wave Basin for Tsunami Research
}

\author{
Solomon C. Yim ${ }^{1}$, Harry H. Yeh ${ }^{2}$, Daniel T. Cox $^{3}$ and Cherri M. Pancake ${ }^{4}$
}

\begin{abstract}
Oregon State is upgrading its multidirectional wave basin under the National Science Foundation Network for Earthquake Engineering Simulation (NEES) program to create a next-generation experimental testing facility for research and practicing engineers. The new facility addresses the unique requirements posed by tsunami researchers, with basin dimensions and wave generation capabilities closely matching the community's vision of the "ideal basin". When completed in 2004, the basin will support high resolution, unprecedented-scale experiments with very dense instrumentation. Researchers will be able to test and validate advanced analytical and numerical models of general environmental fluid mechanics issues in coastal science and engineering including tsunami phenomena induced by sub-sea earthquakes, spanning a full range of ocean, coastal, and harbor studies. An integral part of the upgraded facility is the exploitation of advanced network technologies to allow researchers located at distant sites to play active roles in experiments at the facility, viewing data and images in real time and participating in decision-making. The broader research community will benefit from this large long-wave experiment facility, a comprehensive experiment databank, with specialized interfaces for experiment re-play, searching and browsing archives of past experiments, extraction of experimental data for use in validating numerical models, and a community repository for numerical simulation codes. A summary of the progress on basin building, wave generator and network system design, construction, installation and planned research will be presented.
\end{abstract}

\section{Introduction}

The coastal shorelines of the world are exposed to potentially damaging effects from severe environmental conditions associated with long waves, such as tsunamis and storm surges, which lead to wave run-up and landward inundation. The devastation at any particular location is a function of the velocity, acceleration, and elevation of the water as it interacts with natural and man-made coastal objects. The economic costs of strengthening local infrastructures and evacuating coastal areas are very high; and more importantly, severe tsunamis and storm surges can cause significant losses in human lives. A clear understanding of long-wave runup behavior is critical if we are to develop appropriate warning systems and evacuation strategies. Decisions affecting human safety require systematic methods for evaluating both the long-wave

\footnotetext{
${ }^{1}$ Professor, Civil Engineering Department, Oregon State University, Corvallis, OR 97331

${ }^{2}$ Edwards Chair Professor, College of Engineering, Oregon State University, Corvallis, OR 97331

${ }^{3}$ Director, Wave Research Laboratory, Oregon State University, Corvallis, OR 97331

${ }^{4}$ Professor, Computer Science Department, Oregon State University, Corvallis, OR 97331
} 
events themselves and the wave-structure interaction behavior they are likely to precipitate.

To predict run-up, landward inundation, and wave-structure interaction effects for long waves, the research community turns increasingly to computational models. Physical experimentation is costly and slow, and requires high-resolution, real-time capture of multi-dimensional data. It is also ephemeral, in that there is only one brief opportunity to capture suitable data for a particular run. Numerical experiments offer an attractive alternative. Recently, as a result of a strong effort by the tsunami community, several two- and three-dimensional numerical models have been developed to quantify the interaction of tsunamis with shorelines (Yeh, et al. 1996). The predictive capabilities of these numerical models have not been fully validated, however, due to the lack of suitable field or laboratory data.

Although field measurements of run-up of several recent tsunamis exist, they are insufficient because of the nature of after-the-event field surveys. Very little information about temporal variations can be obtained (except tide gage data) and the data are often extremely spatially sparse. Furthermore, the source of tsunami generation cannot be accurately specified, since any information in deep water is difficult to obtain.

Laboratory experimentation has also been unsatisfactory. In order to accurately scale laboratory long-waves to full size, it is important to preserve the ratios of the most important forces relative to inertia. Most of the laboratory data for long waves available to date are conducted at relatively small scale, however, resulting in nonessential forces (surface tension and viscous forces) being of exaggerated magnitudes to essential forces (gravity, pressure and inertia). A large-scale laboratory facility is essential to obtain usable laboratory data for the validation of numerical models.

In 2000, the US National Science Foundation (NSF) initiated a program called the George E. Brown, Jr. Network for Earthquake Engineering Simulations, or NEES. This is a so-called Major Research Equipment program, intended to provide a national infrastructure of shared-use, next-generation experimental research facilities. The goal of the NEES program is to shift the emphasis of earthquake engineering research, including tsunami studies, from its current reliance on physical testing to integrated experimentation, theory, and model-based simulation.

A key component of NEES is the use of advanced networking capabilities to support teleparticipation - the active, real-time involvement of researchers located at physically distant sites. Further, NEES is a collaboratory, meaning that it integrates not just research activities but also communication and sharing of data in order to support collaborative research. It will include some 20 major earthquake engineering (including tsunami) experimental facilities. They will be linked by NEESgrid - a communications infrastructure based on the high-speed Internet2 network - to facilitate shared use and teleparticipation, and to provide remote access to computational resources. NEES will also provide researchers with remote access to an organized repository of databases, software, and models for use in model-based simulation. This integrated network will facilitate interdisciplinary collaboration among earthquake engineering, tsunami, coastal science and engineering researchers, opening participation to a much broader community than in the past.

In 2000, via a peer-review process, NSF selected eleven host sites as the initial 
"unique, large-scale experimental facilities": a tsunami basin, two shake tables, two geotechnical centrifuges, four structural laboratory systems, and two field measurement systems. The O. H. Hinsdale Wave Research Laboratory at Oregon State University was chosen as the tsunami basin site. The objectives of this paper are to describe the key features of the facility under construction at Oregon State, present a vision for this unique, community laboratory, and discuss the future research collaboration opportunities it will engender.

\section{The Need for Large-Scale Experimental Facilities}

Two- and three-dimensional numerical models are being developed to identify regions along coastlines where long waves can cause damage due to runup and overland flow. Such models are computationally complex and must incorporate movable and deformable surface piercing objects as well as submerged boundaries associated with subaqueous landslides (e.g., Lynett, et al. 2000, Rzadkiewics, et al. 1996; Grilli and Watts 1999). To validate these models, one cannot rely on postevent field observations of runup because of the inability to prescribe the causal waves. Furthermore, field measurements of tsunamis cannot obtain sufficiently detailed spatial and temporal data for model validation. Consequently, laboratory modeling of long waves becomes the expedient alternative to field observation. It must be undertaken at a scale that reproduces prototype behavior, however. In smallscale, free surface-flow experiments, the forces due to viscosity and surface tension can become appreciable compared to inertial, pressure and gravity forces. For ocean wave phenomena, on the other hand, it is generally assumed that gravitational and pressure forces are dominant; this condition will occur only in moderate- to largescale physical models. The scaling dilemma between prototype behavior and small physical models has led the earthquake, tsunami, coastal science and engineering community to conclude that large-scale laboratory experiments are essential.

The problem of scaling is exacerbated by the condition of wave breaking. Long waves shoaling up a coastal slope will reach a water depth where the wave particle velocity will exceed the wave celerity, causing the wave to break. Surface tension affects the shape of the wave and the impact with coastal structures (Peregrine 1995). In some developed coastal areas, it will be desirable to strengthen critical structures so that they can survive some severe events, but evaluating the wave forces requires the proper representation of real fluid properties (Sarpkaya and Isaacson 1981). Drag, inertia and impact force modeling necessitates proper representation of the fluid kinematics and dynamics as well as the coupling mechanism between the relative motion of the fluid and the force on the object. This can be approximated in large physical models, where all forces scale geometrically, facilitating extrapolation of measured results to prototype scale.

Another reason for requiring a large-scale experimental facility in long-wave research is that the horizontal length scale involved in the investigations is usually much larger than the vertical scale. If the wave basin size is limited, it is necessary to employ a distorted model, in which the model to prototype ratio in the vertical direction is different from that in the horizontal direction, resulting in undesirable fluid scaling effects. 


\section{Oregon State's Multidimensional Wave Basin}

As part of NEES, the O. H. Hinsdale Wave Research Laboratory (WRL) will upgrade its existing multidirectional wave basin to become the largest laboratory facility in the Nation for tsunami and coastal engineering research. Since real tsunamis have much greater horizontal dimensionality (tens to hundreds of kilometers long) than depth (just a few kilometers deep), they are categorized as long, or shallow-water, waves. Therefore, the primary component of the new facility is a large-scale, long-wave basin.

Figure 1 shows a plan view of the WRL, including the planned expansion. The existing facility (below the dashed line) includes a $105.6 \mathrm{~m}$ long by $3.7 \mathrm{~m}$ wide by $4.6 \mathrm{~m}$ deep (two-dimensional) wave channel, a $15.4 \mathrm{~m}$ diameter by $1.5 \mathrm{~m}$ deep circular wave basin, and a $26.5 \mathrm{~m}$ long by $18.3 \mathrm{~m}$ wide by $1.5 \mathrm{~m}$ deep multidirectional wave basin. The existing wave generator (Figure 2) in the multidirectional basin consists of 30 wave-board segments. Each paddle is $0.6 \mathrm{~m}$ wide by $1.5 \mathrm{~m}$ high, with a $1 \mathrm{~m} / \mathrm{sec}$ maximum velocity and a maximum stroke of $0.9 \mathrm{~m}$.

The multidirectional wave basin is being expanded to $48.8 \mathrm{~m}$ long by $26.5 \mathrm{~m}$ wide by $2 \mathrm{~m}$ deep. It will be constructed as a reinforced concrete reservoir, with a $0.25 \mathrm{~m}$ wall and floor thickness. Unistrut inserts will be placed in rows at $2.1 \mathrm{~m}$ spacing to affix models, instrumentation, and the wave generator throughout the basin.

The wave generator will consist of 29 wave-board segments, each paddle $0.9 \mathrm{~m}$ wide by $2 \mathrm{~m}$ high. Each wave board will be capable of a $2 \mathrm{~m}$ displacement and a maximum velocity of $2 \mathrm{~m} / \mathrm{sec}$. It will be able to generate a clean solitary wave $0.8 \mathrm{~m}$ high in a water depth of $1 \mathrm{~m}$. Each wave board will be powered by an AC electric motor with a peak power rating of approximately $30 \mathrm{~kW}$. The wave generator will digitally control the paddles on an individual basis, making it possible to generate arbitrary wave profiles and arbitrary wave directions. Control of the wave board will be achieved through displacement and velocity feedbacks. Velocity control will utilize a wave profile measurement at the front of the wave board, comparing it to the desired long-wave profile; board velocity will be adjusted via an algorithm that relates wave profile and board velocity. This velocity control will have the capability of absorbing reflected waves in the basin and optimizing the wave shape beyond that available by means of the displacement control.

The multidimensional long-wave basin will allow repeatable, high-resolution, large-scale experiments with dense instrumentation. The facility is designed to provide a proper environment for implementation of state-of-the-art, non-contact instrumentation (such as optical and acoustic devices) and sensors such as MEMS (micro-electro-mechanical systems). It will enable researchers to validate analytical and numerical models of a variety of long-wave phenomena including longwave/structure interactions for a full range of ocean, coastal, and harbor studies. By controlling changes in bathymetry, surface permeability, roughness, and material erodibility, the effects on long-wave runup and attenuation can be measured.

Although larger wave facilities exist elsewhere, most of them are narrow wave tanks similar to Oregon State's two-dimensional wave channel; for example, the FZK Hanover wave tank is $330 \mathrm{~m}$ long by $5 \mathrm{~m}$ wide by $7 \mathrm{~m}$ high, with a $4 \mathrm{~m}$-stroke 
wavemaker. Wide wave basins similar to the new tsunami basin also exist, but their wave generation capabilities are much smaller. Those facilities are used primarily for studies of coastal wind-generated waves and are not suitable for tsunami studies. The Cedex Basin in France, for example, is $34 \mathrm{~m} \times 26 \mathrm{~m} \times 1.6 \mathrm{~m}$ in size and has a wavemaker with a peak-to-peak stroke of less than $0.6 \mathrm{~m}$, while the US Army Corps of Engineers basin in Vicksburg, MS, is $60 \mathrm{~m} \mathrm{x} 40 \mathrm{~m}$ with a wavemaker stroke of $0.7 \mathrm{~m}$. The multidirectional wave basin at Oregon State will be the first tsunami-scale facility anywhere in the world.

Although tsunamis behave differently from wind-generated sea waves, the basin will be capable of modeling nearshore waves in general. Wind waves generated in deepwater transform to long waves as they approach the shore, due to the decrease in water depth. Consequently, we anticipate that the facility will be attractive to coastal engineering and nearshore-oceanography as well as tsunami researchers.

\section{Advanced Networking and Data Sharing Capabilities}

To broaden the community that can benefit from large-scale, long-wave experimentation, recent advances in computing and networking technology will be leveraged through the ongoing collaboration between WRL and the Northwest Alliance for Computational Science and Engineering (NACSE), also based at Oregon State University. NACSE has developed a comprehensive information architecture that supports the NEES vision of geographically distributed, collaborative use of experimental facilities.

A specially designed teleparticipation environment will allow remote users to take active roles in experiments at the WRL. The instrumentation data acquired during experiments will be accessible through a Web observation interface that exploits high-speed networking capabilities to present information and to relay the responses of remote users back to the facility, both in real-time. Cameras and microphones positioned both above and below the water surface will allow remote participants to select from a number of "views" as the experiment unfolds. These multimedia data streams will be converted to formats suitable for long-distance transmission and synchronized with a range of numerical and graphical representations of the instrument data, making it possible for users to start with an overall perspective on what is occurring, then "narrow-in" on those aspects of most interest to them.

Because they utilize sophisticated equipment and large arrays of instruments, large-scale experiments are time-consuming and costly. One way to reduce requirements for experimentation is to make the results of previous tests available to the general research community. As part of the information architecture, NACSE is developing a Tsunami Experiment Databank that will preserve electronic records of experimental procedures, data, visuals, and results. Intelligent search interfaces will allow prospective researchers to determine if the experiments they are planning replicate or overlap past tests conducted by other investigators. "Replay" capabilities will allow researchers to re-visit experiments even years after they occurred searching, scanning, and fast-forwarding through data streams to find the portions of each experiment that offer the greatest research content.

In addition to reducing the amount of replicated work and increasing awareness of 
the complexity of well-designed long-wave experiments, the Tsunami Experiment Databank will provide direct support for computational modelers. Additional search interfaces will allow them to identify and extract experimental data that can be used to fine-tune and validate numerical simulations. That is, modelers will be able to extract data representing initial conditions, use them as input to the numerical models, and then compare the outputs predicted by the simulations with those actually obtained during experimentation.

The key is to provide flexible mechanisms for locating information about experiments and presenting it in ways that are readily understandable by persons other than the original investigators. Leveraging almost a decade's experience in Web-to-database interfaces that improve the productivity of researchers, NACSE will develop and maintain a databank capable of accommodating at least 15 years of experimental data. A wide variety of data formats will be supported (e.g., video, audio, contact as well as non-contact sensor data, visualizations, comparative or predictive results from computational models, textual summaries, etc.) and be uniformly accessible through self-explanatory, Web-based interfaces. Metadata, the higher-level information necessary to support the exploration of data, will be created to identify experiments that share particular characteristics that led to particular types of results, etc. This will make it possible for users to draw comparisons or analyses that span entire series of experiments, conducted by different researchers and perhaps over widely separated periods of time. Established usability engineering methods the application of human factors research to improve system safety and productivity (Pancake 1998) - will be applied throughout development of the environment to ensure that all user interfaces are simple to understand and operate.

Finally, to enhance the development of analytical models and numerical algorithms and directly support the validation of computer simulation codes, a Webbased numerical codes repository for the tsunami research community will be hosted in conjunction with the Tsunami Experiment Databank. This repository will include two classes of numerical simulation codes. The first consists of "open" numerical codes that owners are willing to make available to the public, such as the TUNAMI code developed by researchers at Tohoku University (Imamura, 1995). The owners or other responsible agencies will furnish periodic updates or maintenance to the codes. The benefit of making these codes more generally available is that more researchers can become familiarized with them, suggest improvements to the codes, and conduct laboratory and numerical experiments to validate them. The second class of codes to be supported in the repository are "proprietary" simulations, offered by commercial entities on a license or fee basis, about which the repository will maintain a directory of information; researchers and practitioners interested in using the codes will need to contact the code owners directly. For both classes of codes, the repository will furnish complete descriptions of the underlying mathematical models, assumptions, numerical algorithms, and range of applicability and limitations. In addition, typical sample results will be posted and updated periodically. A Webbased community forum will also be provided for technical discussions concerning the development and use of numerical simulations in tsunami research. 


\section{Examples of Future Large-Scale Experiments}

The large-scale, multidirectional wave basin will enable a wide range of laboratory experimentation. These will address needs for understanding long-wave phenomena as well as for providing adequate data for model validation in areas such as the following:

- quantitative evaluation of scale effects

- wave breaking and turbulence

- wave-structure interaction

- precise measurements of runup and velocity in a highly three-dimensional flow domain

- tsunami generation and propagation behavior caused by subaqueous landslides

As discussed earlier, a common scale effect is that viscous forces are exaggerated in small models. The effect can be reduced if the model size is increased - although scale effects can never be entirely eliminated - hence a proper scale-effect evaluation is essential for laboratory experiments. Scale effects can be evaluated quantitatively by comparing identical experiments but using a wide range of model scales. Such investigations require a facility like the new WRL expansion, equipped with a precision wave generator and precise basin bathymetry. For example, if the scaling hypothesis is to be examined with runup motion onto a plane beach in a variety of scales (proportional to water depths), wave profiles and velocities must be measured at the same scaled positions relative to the beach toe. Dimensionless profiles and velocities should be identical at the same relative position in the absence of scale effects. Because the distance between the wave generator and the beach toe is physically fixed, the generated wave must be stable to provide identically scaled incident waves. This experiment therefore requires that the wave basin be sufficiently large to cover a wide range of scales, the basin floor be carefully constructed, and the wavemaker system be capable of generating a clean, stable wave such as solitary or cnoidal waves in a variety of water depths.

Another important factor in the scale effects phenomena is associated with wave breaking. Tsunamis often break near the shore, and the approaching flows toward the shore can be violently turbulent. Note that turbulence is a problem that remains to be solved, even at a fundamental level. While turbulence of a simple flow in a small domain can be approximated reasonably well with high-end numerical models, the modeling of turbulence in long-wave phenomena near the shore is far from being even casually approximated. Since turbulence behavior and characteristics are very sensitive to length scales, they cannot be analyzed correctly with small-scale laboratory models. This can be demonstrated by considering the Kolmogorov model (e.g., Landau and Lifshitz 1959). The ratio of the Kolmogorov dissipation length scale to the integral scale of turbulence is proportional to $R^{-3 / 4}$, where $R$ is the Reynolds number. Hence the inertial range of turbulence, and kinetic energy, are sensitive to the scale of the model. Furthermore, turbulence is intrinsically threedimensional; therefore the data taken in a narrow wave tank cast uncertainty on the results. The WRL's new wave basin will be sufficiently wide to play a substantial role in experimental efforts to understand turbulent flow behavior and characteristics. 
Another critical research area is the investigation of wave forces exerted on structures, especially forces associated with breaking and/or broken waves. Impact wave loads on a structure are affected by the scale effect due to viscous and surface tension forces associated with entrapped air-bubbles. Experiments at scales realizable in small laboratory basins produce exaggerated bubble sizes that are almost of the same order of magnitude as that of the impacted body. Because of the size of the expanded facility, it will be capable of testing detailed models in the basin for more accurate measurements and representation of the fluid dynamics.

Investigation of the tsunami forces on structures will be a critical simulation endeavor that will be validated through collaborative experiments involving the new basin and other NEES components such as shake tables, centrifuge equipment, and reaction walls. As an example, suppose a hypothetical earthquake occurs near a port facility where oil storage tanks are located. Deflection and material damage assessment for the tanks will first be made with a NEES shake table, while liquefaction and foundation damage will be investigated utilizing a NEES centrifuge facility. Weakened and partially damaged tanks will be subject to testing in the WRL basin. The tsunami impact force measurements will serve as inputs for further testing of tank damage at a NEES strong-wall facility and to evaluate secondary damage assessment. If a tank material discontinuity is indicated, oil spill patterns will then be analyzed at the WRL to identify potential environmental and fire hazards. Moreover, the tsunami simulation can provide information for water-borne objects that may collide into other structures; those data will be used for further damage simulations. We emphasize that all simulations will be performed in conjunction with complementary numerical and laboratory effort, a mode of investigation made possible by the advanced information architecture provided as part of Oregon State's effort. This type of collaborative simulation capability will be critical to the development of integrated assessment and mitigation strategies for earthquake hazards.

Validating computational models in terms of water-surface elevations alone is insufficient. In fact, since the water-surface elevation is obtained by integration of the hydrodynamics equations, it is relatively insensitive to errors in other parameters. For adequate model validation, it is essential that accurate velocity-field data be provided. Predicting coastal long-wave kinematics is difficult in practice, however, because many coastal bathymetries and topographies are highly complex and threedimensional. A highly three-dimensional bathymetry is needed as a benchmark for model validation, which means that dense instrumentation patterns and accurate data for water-surface elevations and the velocity field must be obtained.

The new WRL basin can also be used to explore the mechanisms of landslide wave generation in collaboration with the geotechnical engineering community. Landslide generated tsunamis are known to cause significant impacts locally. While these events have been documented in the past (such as the Lituya Bay event in 1958, discussed in Miller 1960), the topic has received more attention recently due to the 1998 PNG event (e.g., Matsuyama, et al. 1999; Tappin, et al. 2001; although the cause of this tsunami is still controversial). The topic involves many uncertain factors including the behavior of the landslide itself. The generated wave is highly three-dimensional and dispersive (wavelength is not as long as that of tsunamis of 
tectonic origins), calling for the specialized capabilities of the new facility.

The experimental programs discussed previously are visually dynamic flow phenomena (e.g., wave breaking and wave interaction with structures). Nevertheless, a large-scale three-dimensional basin such as the new WRL facility is also useful for investigating very subtle tsunami induced flow phenomena. A typical tsunami generated from tectonic origins has a large horizontal scale (tens to hundreds of kilometers long), but very small wave amplitude at the origin (a few meters). Such a linear and very weakly dispersive wave is extremely difficult - almost impossible - to simulate in a laboratory facility and is not visually dynamic: there is no splash and no noise. Yet this is a typical tsunami characteristic. When such a tsunami enters a continental shelf, the wave may experience soliton fission (Madsen and Mei 1969). Further, the wave becomes more nonlinear near the shore and where runup occurs onto the beach. At least a portion of such a transformation process can be investigated using the new basin. It requires the precise motion control of the wavemaker to generate a clean, linear and very long wave; the movement is slow and short. The generated wave in the laboratory may be $10-20 \mathrm{~m}$ long and less than $1 \mathrm{~cm}$ in wave amplitude, so water-surface elevations must be measured accurately to submillimeter precision. Since it is a long wave, the basin floor and beach must also be constructed precisely. Clearly, fundamental simulations such as this, while key to understanding the behavior and characteristics of real tsunamis, will only be achievable in the unique large-scale facility at Oregon State University.

\section{Future Collaboration Opportunities}

Oregon State University's tsunami basin facility will become available as a NEES equipment site for research use by October of 2004 and will be operational for at least ten years. Currently, NSF is supporting the development of a Consortium that will manage the operation and use of all the NEES equipment facilities. It is envisioned that the tsunami research community will meet periodically to identify critical research topics and to establish research agendas and priorities for tsunami research in general, and experimental research in particular. The community will submit their findings to the Consortium, which will then determine research funding for tsunami experiments at the WRL facility. Researchers in coastal science and engineering and other related communities are encouraged to take a similar approach for the planning, development, and prioritizing of experiments, in order to take advantage of this unique laboratory for long and short-wave research. The Oregon State facility is a community driven, community operated, and partially NSF supported research tool. Research collaboration will be essential to ensure that the WRL basin provides maximum benefit to the research community.

\section{Summary and Concluding Remarks}

A unique large-scale, three-dimensional, long-wave facility is under construction at the O. H. Hinsdale Wave Research Laboratory at Oregon State University. The facility will enable a new genre of research studies on long waves, including tsunamis and storm surges. It will also serve to explore nearshore wave characteristics and behavior for coastal engineering problems. Because of its large surface area, great 
depth, and precision wavemaker, detailed experiments for critical long-wave problems (e.g., scale effects and turbulence) can be conducted. By accommodating a full range of state-of-the-art instrumentation, the basin will also provide the analytical and numerical modeling community with a high-resolution tool for validating predictive models.

Through the application of advanced networking and computing technologies, the facility will be available to the international research and development community, including those at remote locations. The Tsunami Experiment Databank will make it possible to share data, visuals, and results with research collaborators in real time. The broader community will be able to benefit from specialized capabilities for experiment re-play, searching and browsing the archive of past experiments, extraction of data from experiments for use in validating numerical models, and access to numerical simulation codes. By providing comprehensive support for the tsunami research community, the facility will promote multidisciplinary and collaborative research with researchers across the globe.

\section{Acknowledgement}

We gratefully acknowledge the financial support by the National Science Foundation (CMS-0086571) for upgrading and expansion of the Oregon State tsunami wave basin and the 2001 community workshop on research using the NEES tsunami facility (CMS-0116898), and by the Office of Naval Research for the original construction of the multidirectional wave basin and continuing research (N00014-86K0687 and N00014-92-J1221).

\section{References}

Grilli, S.T. and Watts, P. (1999). "Modeling of waves generated by a moving submerged body: applications to underwater landslides." Engineering Analysis with Boundary Elements, Vol.23, No.8, pp. 645-656.

Imamura, F. (1995). "Tsunami numerical simulation with the staggered leap-frog scheme," manuscript for TUNAMI code, School of Civil Engineering, Asian Inst. of Tech., 45 pp.

Landau, L.D., and Lifshitz, E.M. (1959). Fluid Mechanics, Pergamon Press. 536 pp.

Lynett, P. J., Liu, P. L.-F., Losada, I. J. and Vidal, C. (2000) "Solitary wave interaction with porous breakwater", J. Waterway, Port, Coastal and Ocean Engrg., ASCE, Vol.126, pp. 314-322.

Miller, D.J. (1960). "Giant waves in Lituya Bay, Alaska." U.S. Geological Survey Professional Paper 354-C, 83 pp.

Matsuyama, M., Walsh, J.P., Yeh, H. (1999). "The effect of bathymetry on tsunami characteristics at Sissano Lagoon, Papua New Guinea," Geophysical Research 
Letters, Vol. 26, No. 23, pp. 3513-3516.

Pancake, C.M. (1998). "Improving the usability of numerical software through user-centered design," in The Quality of Numerical Software: Assessment and Enhancement, ed. B. Ford and J. Rice, Chapman \& Hall, London.

Peregrine, D.H. (1995). "Water wave impact on walls and the associated hydrodynamic pressure field," Wave Forces on Inclined and Vertical Wall Structures, Kobayashi and Demirbilek, eds., ASCE, pp. 259-281.

Rzadkiewics S. A., Mariotti, C., and Heinrich, P. (1996.) "Numerical simulation of submarine landslides and their hydraulic effects", J. Waterway, Port, Coastal, and Ocean Engrg., ASCE, Vol.123, pp. 149-157.

Sarpkaya, T. and Isaacson, M. (1981). Mechanics of Wave Forces on Offshore Structures, Von Nostrand Reinhold Co., New York, 651 pp.

Tappin, D.R., Watts, P., McMurty, G.M., Lafoy, Y., Matsumoto, T. (2001) "The Sissano, Papua New Guinea tsunami of July 1998 - offshore evidence on the source mechanism," Marine Geology, Vol. 175, pp. 1-23.

Yeh, H., Liu, P., and Synolakis, C. (1996). Long-Wave Runup Models, World Scientific Publishing Co., Singapore, 403 pp. 


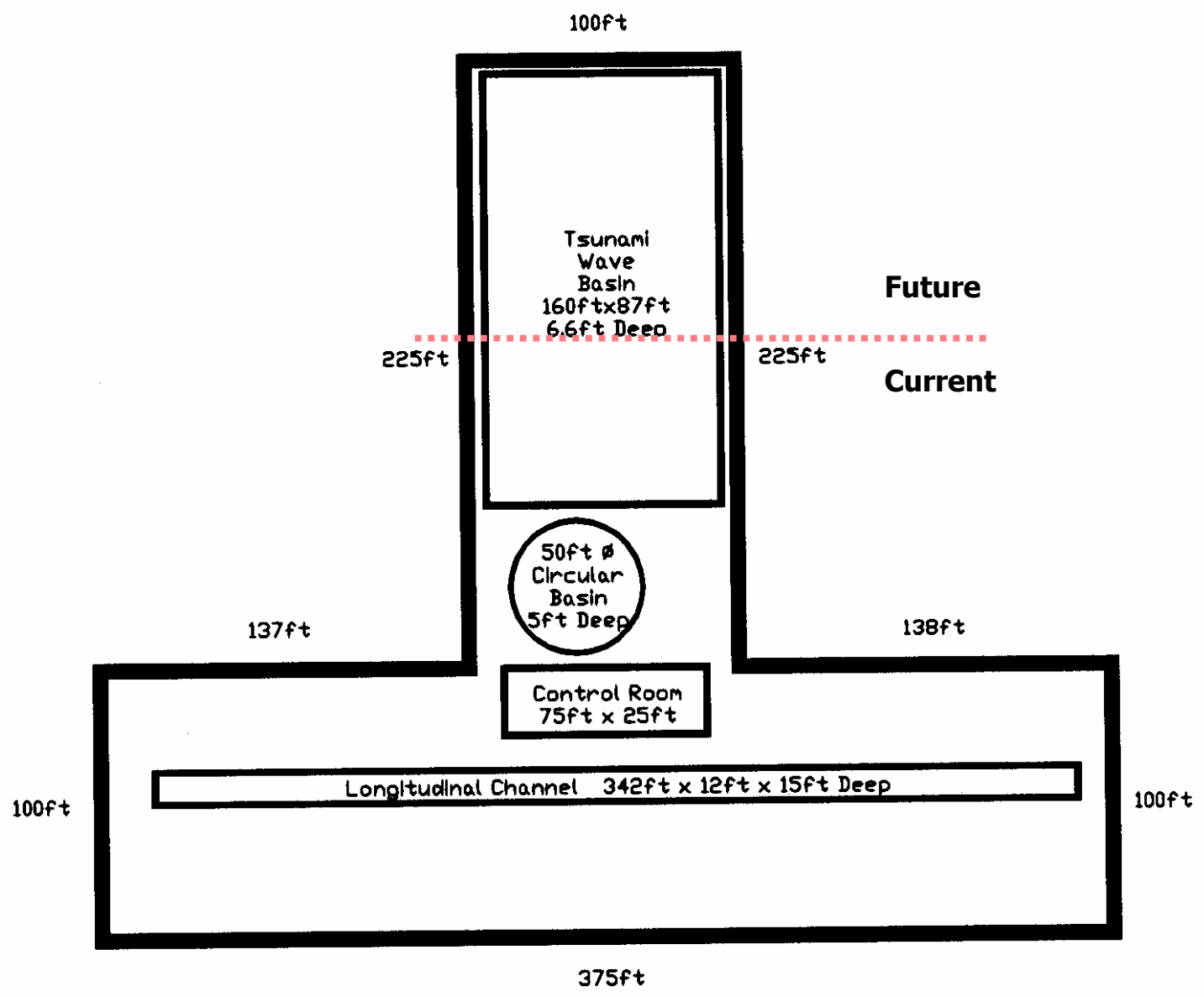

Figure 1. Plan view of Oregon State University’s Wave Research Laboratory. 


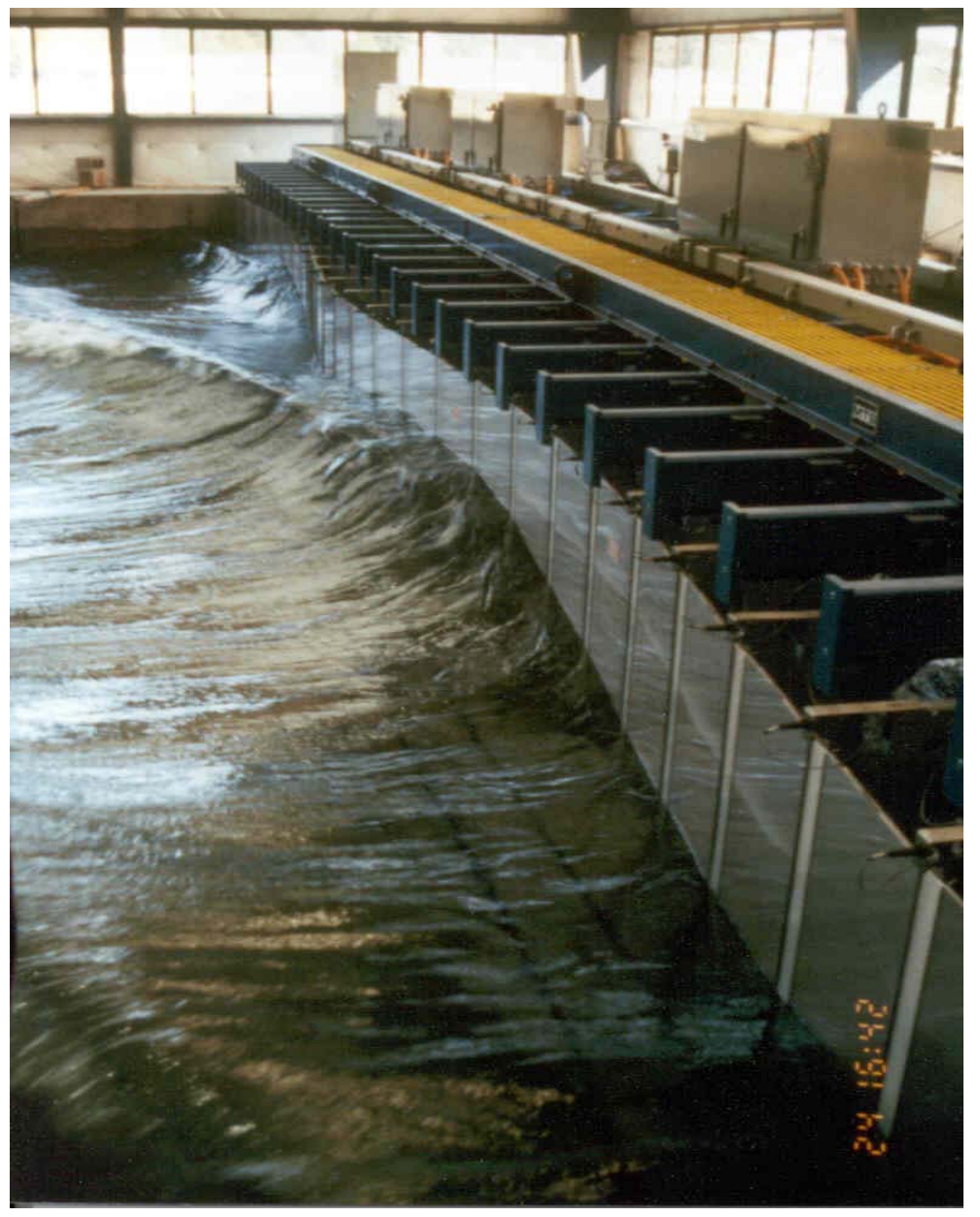

Figure 2. Existing Multidirectional Wave Generator. 